Published in final edited form as:

Curr Opin Immunol. 2017 August ; 47: A1-A2. doi:10.1016/j.coi.2017.09.001.

\title{
Editorial overview: Vaccines
}

\author{
Ross M Kedl and Robert Seder
}

Vaccination is one of the most successful medical interventions in recorded human history, transforming global health in a way comparable to sanitation and water infrastructure. The first implementation of vaccination, known as variolation (purposeful exposure of healthy people to infected tissue), was likely practiced in China around 900AD, but it was Jenner and Pasteur whose empiricism and use of the scientific method forged our modern use and understanding of vaccination. While these guiding principles are still applicable today, recent advances facilitate a far more comprehensive assessment of humoral and cellular immune responses, providing the foundation for a more rational approach to vaccine design and development. This has effectively revolutionized the application of immunological principles to the design and development of novel vaccines. In the last 20 years, whole families of novel innate receptors have been identified and the immunological consequences of their signaling pathways elucidated. The potency of these innate receptors for raising robust innate inflammatory responses when triggered by their identified ligands has fueled much optimism for finally achieving vaccine solutions to difficult infectious pathogens and cancers. Of course, early optimism is often uninformed, our predictions being continually revised by the successes and failures that happen along the way. The articles in this Vaccines issue document the successes, failures, and lessons learned, ultimately lending their expertise to the future of vaccination.

While there have been great advances in successful vaccines against a number of bacterial and viral infections, there still remains tremendous needs and opportunities in several important clinical areas. Accordingly, morbidity and mortality from HIV, malaria and tuberculosis remain unacceptably high around the world. There remain several scientific hurdles that limit vaccines against such infections. A vaccine against HIV will probably require both the capacity to induce a robust cellular response (Picker) and/or the induction of a broadly neutralizing antibodies (Torres), demanding advances in immunogen and adjuvant formulation and how these influence the priming of Tfh and B cells (Crotty). A vaccine against tuberculosis has been similarly difficult to achieve, though new advances in vaccine vectors and adjuvants suggest a solution to this global disease may be closer than ever (Kaufmann, Izzo). Adjuvants have always had a critical role in improving vaccine efficacy, and modern cellular and molecular tools have provided important insights into both old and new adjuvants and their mechanisms of action (Bhardwaj, Marrack, O'Hagan, Lore, Mitchell). Finally, it has long been known that $\mathrm{T}$ cells can play a critical role in mediating anti-tumor immunity, a fact highlighted in the last several years by the enhancement of host immune responses through blockade of checkpoint inhibitors such as PD-1/PD-L1 and CTLA4. While clinical responses to these treatments have been dramatic and encouraging, the most successful achieve $\sim 40 \%$ efficacy, leaving much room for improvement. This has created a renewed interest in the pursuit of vaccines capable of further enhancing CTL and Th1 $\mathrm{T}$ cell responses, a more difficult goal than inducing antibody production. Advances in 
tumor neo-antigen identification, adjuvant biology, and targeting of specific molecules involved in adaptive responses provide strong support for the pursuit of a variety of therapeutic vaccine approaches in the clinic (Overwijk, Bullock, Celis, Slingluff).

For this Vaccines issue, we enlisted a group of contributing authors that have extensive scientific expertise in the development of preventive and therapeutic vaccines for infections and cancer respectively. These authors have a deep understanding of the underlying immunological mechanisms driving effective vaccination as well as informed opinions on clinical translation. Given their expertise, we allowed the voice and opinion of each contributor to come through essentially unfiltered by editorial input. The normal process in editing review articles is to send each out for 'expert review' by other knowledgeable contributors to the field. While reasonable for other review journal formats, we thought the notion of having other experts give their opinions about the expert opinions expressed by our authors was not only circular but counter-productive. Thus, we limited our editorial influence on each article to facilitating clarity. Finally, as the descriptions above highlight, some topics are covered by multiple articles; for example, molecular adjuvants, cancer vaccines, vaccines against tuberculosis, etc. Having overlap allowed for both consensus and diversity of opinion in these complex areas of vaccine research. We thank all of the authors for the time and scholarly attention they devoted to each article and hope that it will guide current and future investigators in their efforts to make successful vaccines. 